\title{
Coulisses
}

Revue de théâtre

12 | Printemps 1995

Varia

\section{Partage de Midi : de la vie à la fiction}

\section{Gérald Antoine}

\section{OpenEdition}

Journals

Édition électronique

URL : http://journals.openedition.org/coulisses/3273

DOI : $10.4000 /$ coulisses.3273

ISSN : 2546-9460

\section{Éditeur}

Presses universitaires de Franche-Comté

\section{Édition imprimée}

Date de publication : 1 mai 1995

Pagination : 10-15

ISSN : 1150-594X

\section{Référence électronique}

Gérald Antoine, «Partage de Midi : de la vie à la fiction », Coulisses [En ligne], 12 | Printemps 1995, mis en ligne le 15 mars 2019, consulté le 31 octobre 2019. URL : http://journals.openedition.org/coulisses/ 3273 ; DOI : 10.4000/coulisses.3273

Ce document a été généré automatiquement le 31 octobre 2019

Coulisses 


\title{
Partage de Midi : de la vie à la fiction
}

\author{
Gérald Antoine
}

1 Le sous-titre déborde largement le titre : quel est l'écrivain dont les fictions ne se sont pas nourries de souvenirs demeurés vivants? - Je songe à un exemple tout neuf, poignant, qui fait du bruit, d'analyse du phénomène : Jorge Semprun, L'Ecriture ou la vie. 1

Mais il est un danger auquel on s'expose lorsqu'on veut évoquer en un temps restreint la manière dont un artiste a transposé tel épisode de sa vie en une œuvre littéraire.

Là encore un exemple récent m'a frappé. Marc Lambron, auteur de l'Eil du silence ${ }^{2}$, calque romanesque de la vie extraordinaire, et si incroyable qu'elle paraît inventée, d'une femme photographe engagée dans l'aventure surréaliste, maîtresse de Man Ray et de bien d'autres, était convié l'autre jour à parler de son livre et à décrire le passage du réel à l'interprétation romanesque. Eh bien l'auteur nous a raconté pendant une heure l'existence de cette femme, mais n'a pas dit un mot sur son travail, à lui, de métamorphose littéraire. Quelle leçon pour moi !

J'évoquerai donc, à égalité de temps, le partage de vie entre Paul claudel et Rosalie Vetch puis Lintner - et Partage de Midi, transposition théâtrale que le poète a opérée à partir de son expérience de la passion.

\section{I. - Partage de vie}

2 En fait il fut double : d'une part avec Dieu, de l'autre avec Rosalie.

Avec Dieu d'abord. L'événement fondateur, ce fut l'«illumination » de Notre-Dame surgissant aussitôt après la lecture des Illuminations de Rimbaud.

Suivirent quatre années entre lumière et ténèbres jusqu'à la vraie « conversion » : 1890 . C'est l'année où Claudel est reçu au concours des Affaires Étrangères. Après quoi on le trouve en poste à Paris, puis en Amérique (New York, puis Boston) où il écrit l'Échange, dont le thème et les quatre personnages sont déjà bien proches du quatuor de Partage de Midi. Vous rappellerai-je à ce propos deux textes instructifs ô combien : 
Antérieurement aux expériences redoutables que j'ai vécues plus tard, je les décrivais d'avance, avant de les avoir subies ${ }^{3}$ (Mémoires improvisés, p. 30)

L'Artiste est le contemporain de toute sa vie. Les événements dont il n'a pas le souvenir, il en a le pressentiment. (Ma sœur Camille. Euvres en prose, p. 284) ${ }^{4}$

Viennent ensuite les trois séjours en Chine : 1895-1899 ; 1901-1905 ; 1906- 1909 - ce qui fait quatorze ans au total. Celui de 1901-1905 est au cœur de notre sujet. C'est alors que se situe le second partage, indissociable du premier : le partage avec la Femme.

Ce fut - le mot s'impose - sa « Saison en Enfer ». Mais il ne faut surtout pas oublier son prélude mystique : les deux retraites à Ligugé au cours de l'été puis de l'automne 1900 : Claudel se croit confusément destiné à une vocation de clerc et même de moine. Nulle réponse claire ne lui vient de Dieu.

Le 15 octobre 1900 il s'embarque à Marseille pour la Chine. Sur le bateau il rencontre ou plutôt retrouve Rosalie Vetch accompagnée de son mari et de ses enfants. Parmi les passagers figure un personnage d'un relief particulier: un certain Castanier, fonctionnaire colonial qui rejoint son poste au Cambodge. C'est lui qui pousse Claudel vers Rosalie et qui lui conseille d'accueillir à Fou-tchéou le ménage sous son toit.

A Fou-tchéou, Claudel aide efficacement Francis Vetch dans ses entreprises commerciales. Il l'encourage dans la prospection des mines du Fou-kien. Pendant ce temps il devient l'amant de Rosalie et la persuade de confier ses enfants à une gouvernante. Rosalie devient à la fois maîtresse de l'hôte et maîtresse de maison : en 1903 le faux ménage Claudel reçoit joyeusement le faux ménage Berthelot : aube d'une amitié capitale.

4 En 1904 rumeurs et dénonciations parviennent au Quai d'Orsay qui dépêche deux enquêteurs. Ils concluent à un non-lieu, mais il est conseillé à Claudel de faire partir Rosalie et de demander pour lui-même une mutation. Le $1^{\mathrm{er}}$ août 1904, Rosalie quitte Fou-tchéou, alors qu'elle est enceinte de Claudel. Elle rejoint l'Europe via le Canada.

Du bateau elle écrit à "son petit consul ». Puis silence - un silence qui durera treize ans : elle ne lui répond plus et lui renvoie ses lettres. Une tante de Rosalie, religieuse, apprend à Claudel que Rosalie vit auprès d'un autre homme et qu'elle a mis au monde une fille.

Désespoir de Claudel, rentré en France au printemps 1905.

Accompagné de Francis Vetch il poursuit en vain la fugitive. Il connaît alors deux tentations, l'une destructrice : le suicide, l'autre salvatrice : l'écriture. De celle-ci va naître Partage de Midi $i^{5}$ en quatre étapes. Fin 1904 - début 1905, manuscrit «A " interrompu par la «nouvelle de l'horrible trahison». A partir du 26 mai 1905, manuscrit « B » très partiel. Du 30 août au 21 novembre 1905, manuscrit « $C$ » complet. Enfin dernier manuscrit porté aux éditions de l'Occident le 27 février 1906, jusqu'ici non retrouvé.

Je reviens aux événements extérieurs: Claudel se fiance, puis se marie avec Reine Sainte-Marie Perrin, décembre 1905-mars 1906. Le jeune ménage part aussitôt pour la Chine : Pékin, puis Tientsin, retour en France en août 1909.

Claudel occupe cinq postes en Europe: Prague (1910-1911) ; Francfort (1911-1913); Hambourg (1913-1914) ; Paris avec repli sur Bordeaux (1914-1915); Rome (septembre 1915-novembre 1916).

En février 1917 il est nommé Ministre de France à Rio qu'il rejoint en compagnie de Darius Milhaud. Le 4 août 1917 lui parvient une lettre de Rosalie qui le bouleverse (j'ai été autorisé à reproduire des extraits de sa réponse). L'auteur du Soulier de Satin en fera « la lettre à Rodrigue ». Je puis vous révéler au passage qu'outre deux des « lettres 
affreuses » envoyées par Claudel à Rosalie en 1905, environ cent-quatre-vingts autres sont conservées dans un Fonds inaccessible au public avant le début du siècle prochain.

A Rio Claudel sera vivement séduit - mais tenu à distance - par la belle et pétulante Audrey Parr, alias Margotine.

En 1918 il est muté à Copenhague où il sera en poste d'août 1919 à mars 1921. Nous retiendrons surtout deux séjours qu'il fait en décembre 1920 et mars 1921 à Londres où il « retrouve » Rosalie et découvre leur fille Louise :

Les circonstances ont permis qu'entre les deux partenaires de Partage de Midi, une "retrouvaille», on peut dire, ait eu lieu, une rencontre, une explication, et

finalement un apaisement dans un sens élevé6(Mémoires improvisés, p. 269)

Il est ambassadeur au Japon de septembre 1921 à juin 1926, avec une coupure d'un an, conséquence de l'apocalypse du $1^{\mathrm{er}}$ septembre 1923 . Je note, en marge de cette étape, deux faits : c'est Rosalie qui accompagne Claudel jusqu'à la gare lors de son départ pour le Japon; lors du séisme il perd la $3^{\text {ème }}$ Journée du Soulier de Satin (qu'il achèvera en 1924) et "une dizaine de poèmes qui contiennent les thèmes du Partage » (lettre à Jacques Rivière).

De février 1926 à avril 1933 il est ambassadeur à Washington, avec un congé de mai à octobre 1930. À Washington, il éprouve une amitié amoureuse pour Agnès Meyer, à laquelle il lui arrive d'écrire journellement. Résultat : le ton des lettres à Rosalie change et leur rythme se ralentit.

De mai 1933 à juin 1935 il occupe son dernier poste d'ambassadeur à Bruxelles. Il y nourrit une amitié très affectueuse pour la Comtesse Lippens et une admiration fervente pour la Reine Elisabeth.

Claudel connaît pour finir vingt années de retraite: 1935-1955. La guerre et l'occupation le confineront la plupart du temps à Brangues où il donne le meilleur de ses forces à d'extraordinaires commentaires de la Bible. Autre fait majeur sur le plan de l'art: les fruits de sa découverte (dès 1937) de J.-L. Barrault d'où va sortir la mise en scène du Soulier de Satin en novembre 1943, de Partage de Midi en décembre 1948, de L'Echange en décembre 1951, de Christophe Colomb en décembre 1954, pour ne parler que des créations abouties de son vivant.

\section{II. - Partage d'écriture dramatique}

6 Ne visant nullement les effets de surprise, je vous propose d'inventorier ce qui, dans le drame écrit, est emprunté d'une part au réel, plus ou moins transposé, d'autre part à l'imaginaire, mis en œuvre pour prendre le relais du réel. Après quoi, plutôt qu'une conclusion, je souhaiterais esquisser une interprétation de cette dualité dans l'ordre thématique.

\section{A. - Ce que le drame emprunte de plus près à la réalité}

7 On peut dire en bref : essentiellement les deux premiers Actes. Cf. sa lettre à Massignon du 19 novembre 1908: " les deux premiers actes de Partage ne sont qu'une relation exacte de l'aventure horrible où je faillis laisser mon âme et ma vie ».

Une lettre à Jammes du 19 septembre 1905 nuançait en apparence davantage : ce " drame qui n'est autre que l'histoire un peu arrangée de mon aventure » [aventure dans laquelle, à cette date, il se débat encore]. 
Les mots « un peu arrangée » s'appliquent surtout, en fait, à l'Acte III.

Considérons d'abord les personnages. Dans le manuscrit «A ", ils sont trois, comme ils l'étaient jusqu'au départ de Rosalie : elle - lui - le mari. À partir du manuscrit «B " s'ajoute, comme dans la réalité découverte après coup par Claudel, un second amant. L'auteur dramatique s'est toutefois permis une variation de son cru: le mari, peint d'abord comme un robuste bourguignon, est devenu un « maigre Provençal »!

Les lieux scéniques accusent une triple fidélité au réel : les lettres de Rosalie à sa mère m'ont permis de la vérifier pour l'intérieur de la maison; Gadoffre l'avait déjà constatée pour le paysage de Fou-tchéou vu de la maison; enfin le « jardin maudit » est un rappel appuyé du cimetière et de la tombe en $\Omega$ où les deux amants aimaient à se retrouver.

Quant à l'action, je vous renvoie à l'analyse qu'en fit Claudel dans la Préface de 1948 : le drame écrit, à l'image exacte du drame vécu, réunit deux données: l'adultère, et la lutte entre la vocation religieuse et l'appel de la chair. Détaillons quelques traits :

Sur le bateau, la «scène » que Mesa fait à Ysé et que celle-ci raconte à Amalric est un épisode vécu. En témoignent une confidence de Claudel à Barrault et l'une de ses lettres à Rosalie.

Le récit que Mesa fait à Ysé de l'échec de sa vocation monastique - des recoupements me permettent de l'affirmer - est plus «vrai», plus sincère que les relations autobiographiques que Claudel en a données.

A Fou-tchéou même : tout le contenu de l'Acte II et quelques éléments de l'Acte III sont un écho direct de la réalité. Je retiendrai dans l'Acte II : l'entente cordiale entre Mesa et De Ciz. La manière dont l'amant favorise l'éloignement du mari. Les orages, les cruautés et, pour finir, l'échec de la passion: Mesa ne sait pas se donner; Ysé est la séductrice porte-malheur, la femme fatale au sens antique.

À l'Acte III : les « lettres affreuses » de Mesa, comme la «lettre affreuse » que Mesa a reçue d'un tiers. Le silence total d'Ysé. La permanence de la passion et sa «transfiguration » selon le credo de Mesa.

\section{B. - Ce que le drame apporte d'invention}

8 En bref, la majeure part de l'Acte III relève de l'affabulation.

Le lieu même de l'action est à demi imaginaire. D'ailleurs, d'une version à l'autre, l'auteur a hésité : la «maison dans l'ancien style colonial» de la première est, je le répète, très proche du modèle, mais le « temple confucéen... pas mal endommagé » de la seconde s'en éloigne. Autrement dit, la part de l'imaginaire progresse de l'une à l'autre.

L'action elle-même est totalement recréée, même si certains éléments - à commencer par le retour final d'Ysé vers Mesa - nous apparaissent aujourd'hui comme l'anticipation transposée d'une réalité future.

Faisons donc l'inventaire des choses inventées: le retour d'Ysé «dans le sud de la Chine ». - L'irruption de Mesa dans le second ménage d'Ysé. - Le pugilat (qui n'est même plus du drame, mais plutôt du mélodrame !). - L'abandon par Ysé de son second amant. - La mort de leur enfant. - La mort atroce de son mari. - La mise en marche de la machine infernale, et la mort imminente d'Ysé et de Mesa dans l'explosion de leur repaire... Cela fait, notons-le, beaucoup de morts (quatre) comme dans les tragédies antiques. Cela fait aussi une assez belle dépense d'imagination concentrée sur un seul Acte. 
Il importe toutefois de souligner ici une intime et mystérieuse imbrication de la fable et d'une réalité ressentie ou pressentie. J'en citerai cinq exemples :

Le Cantique de Mesa, cet apogée du drame en forme d'oratorio est à coup sûr un prodige d'imagination quant au fait; mais il est intensément fidèle à la réalité des sentiments éprouvés par Claudel.

Mesa et Ysé se retrouvant à l'heure du dénouement, c'est de l'invention - mais elle préfigure la «retrouvaille » très réelle de Paul et de Rosalie dans la suite de leur destinée.

Ysé se convertit de manière surprenante dans le drame - mais Rosalie, de même, se convertira dans la vie.

Ysé appelle Mesa à se donner pour de vrai : c'est une des clés du drame - mais la correspondance de 1905 est là pour nous prouver que le plus puissant grief de Rosalie se situa précisément de ce côté.

Enfin Mesa et Ysé refusant au terme une union sur terre au profit de noces dans l'éternité sont à l'exacte image de ce que proposera Paul à Rosalie dans plusieurs de ses lettres (celle entre autres du 10 février 1921) 7 .

\section{C. - Tentatives d'interprétation}

9 Tout est loin d'être parfaitement clair lorsque l'on veut serrer de près le texte de Partage de Midi. Je m'en tiendrai pour cette fois à l'approfondissement de deux aspects thématiques sans nul doute fondamentaux.

Claudel nous met sur la voie du premier à travers plusieurs textes. Parmi eux je citerai un entretien avec André Bourin paru dans les Nouvelles Littéraires du 23 décembre 1948 :

Partage est le drame de la passion. La passion préparée, épousée, elle aboutit. Elle aboutit à la souffrance. Pas seulement à la souffrance. À la mort. - Pas seulement à la mort.

Puis une phrase tirée de son dernier grand texte, Conversation sur Jean Racine $e^{8}$ : "Le drame humain [...] n'est pas complet, tant qu'un élément surhumain ne vient pas s'y mêler ».

Mauriac était le mieux préparé à entendre ce mystère. Il en témoigne avec éclat au lendemain de la création de la pièce au Théâtre Marigny (Figaro du 20 décembre 1948) :

Depuis que j'aime cette pièce, depuis que je l'ai lue à mon aurore, de plus hauts chefs-d'œuvre, même Tristan, m'apparaissent comme bouchés. Même Phèdre : Dieu y est présent, mais c'est le Dieu théologien, le Dieu implacable, non celui à qui Mesa mourant ose crier: "Si vous avez aimé chacun de nous comme j'ai aimé cette femme... »

11 Seconde perspective thématique à explorer plus à fond : ce qu'il ne faut pas craindre, à mon sens, de dire, c'est que trois difficultés au moins empêchent le spectateur non prévenu d'entrer pleinement dans le sens du $3^{\text {ème }}$ Acte.

D'une part la dernière scène de Partage n'est pas son véritable dénouement. Elle ne fait que l'annoncer. Et ce dénouement est double : c'est d'abord la mort imminente des deux amants qui est dérobée à nos yeux, et c'est ensuite leur rédemption, fruit de cette mort expiatrice. A cet égard une phrase de la lettre de Paul à Rosalie, du 10 février 1921, est parfaitement éclairante : « tout entre nous expié, pardonné ».

D'autre part il y a, dans le contenu même de l'intrigue un rude obstacle à surmonter, à savoir la somme et la gravité des péchés commis, "partagés » par les deux amants: comment peuvent-ils être assurés (et nous avec eux) qu'ils seront pardonnes? - 
L'Évangile apporte sa réponse : « Là où le péché abonde, la Grâce surabonde ». Notonsle d'ailleurs au passage : l'affirmation attribuée à Saint Augustin: Etiam peccata, donnée comme épigraphe au Soulier de satin et que l'Ange, (répondant à la question de Prouhèze : «L'amour hors du sacrement n'est-il pas le péché ? ») traduit ainsi : « Même le péché ! Le péché aussi sert », - cette proclamation convient beaucoup mieux à Partage de Midi où les protagonistes cèdent à la tentation, qu'au Soulier de satin, où ils $\mathrm{y}$ résistent.

Dans le Partage Ysé comme Mesa confesse sa perdition. C'est même elle, la plus grande pécheresse, qui amène Mesa à reconnaître sa propre part de faute : l'avare, l'égoïste foncier qu'il est n'a pas su se donner. Il a péché non point par, mais contre l'amour entendez: l'amour-agapê dont l'amour-éros n'est qu'un spécieux reflet. Cependant, c'est à lui, le prêtre en esprit, qu'il échoit en retour d'inviter Ysé à recevoir les paroles sacramentelles : « ...je consens à toi, Ysé !» (sacrement du Mariage). « Voyez, mon Dieu, car ceci est mon corps » (parole de la Consécration).

C'est à lui aussi qu'appartiennent les derniers mots :

Souviens-toi, souviens-toi du signe (...)

L'homme dans la splendeur de l'août, l'Esprit vainqueur dans la configuration de Midi.

Entendez bien : leur figure humaine, «visage mortel », va se dissoudre; ils renaîtront trans-figurés.

Ce dénouement me fait penser (cela, je ne le dis pas dans l'édition de Partage, mais je le soumets à votre réflexion) à la fin de la première et surtout de la deuxième Partie de Faust, n'en déplaise à Claudel, pourfendeur de Goethe ! - Quand Faust vient rechercher Marguerite, à la fin de la première Partie, elle ne lui parle que de l'expiation de sa faute et de sa mort. Elle repousse Méphisto - et lorsque celui-ci déclare : «Elle est jugée » (Sie ist gerichtet), une Voix d'en haut répond: "Elle est sauvée ( Sie ist gerettet). Ainsi trouvons-nous déjà réunis les deux temps du thème fondamental: péché suivi d'expiation sur terre; absolution dans l'au-delà. Mais ce n'est pas tout : à la fin de la deuxième Partie nous retrouvons Marguerite. Et cette fois c'est elle, la pécheresse pardonnée, qui, aux pieds de la Mater gloriosa, va demander et obtenir le salut de Faust qu'elle n'a pas cessé d'aimer. C'est le second degré du même thème que réorchestrera Claudel : rédemption par l'Amour et transfiguration dans l'outre-tombe.

Venons-en, pour finir, à la troisième occasion d'achopper. C'est un collègue et ami bisontin, claudélien de haut renom, Michel Malicet, qui me l'a récemment signalée. Elle peut se résumer ainsi : comment concilier l'annonce finale d'une "transfiguration » ( «...l'Esprit vainqueur dans la transfiguration de Midi!») avec celle, faite un instant auparavant, par le même Mesa : « ...au-delà de la tombe j'entends se former le clairon de l'Exterminateur »?

La relecture scrupuleuse du contexte interne et externe procure la réponse tout en accentuant l'apparence de contradiction.

Contexte proche d'abord. Dans la même dernière scène de Partage Mesa joint " d'une part au-delà de la tombe [...] le clairon de l'Exterminateur » - «Et d'autre part [...] tous les événements de ma vie à la fois devant mes yeux [qui] se déploient comme les sons d'une trompette fanée ". Ainsi l'anéantissement de l'au-delà répond à l'anéantissement de l'en-deçà. Mais à ces accents deux fois nihilistes Ysé rétorque violemment: «Que parles-tu de la trompette perçante ? lève-toi, ô forme brisée... » et toute la suite est la réplique victorieuse du chant libérateur.

Notez en outre la didascalie, plus révélatrice encore, de la $2^{\text {ème }}$ version pour la scène : 
«On ne voit plus que la main lumineuse de Mesa, levée, qui à elle seule meuble toute la cavité de la scène ».

Contexte externe ensuite - celui-ci vraiment fait pour achever de nous troubler. Je l'emprunte à l'Antistrophe III de La Muse qui est la Grâce :

...Voici l'œuvre d'Août, voici l'extermination de Midi.

Où donc est la vérité : du côté de l'extermination, ou du côté de la transfiguration ? Un titre pirandellien nous fournirait la réponse : «A chacun sa vérité ». Prenons bien garde en effet : le héros de la IV ème Ode, comme le héros de Partage aussi longtemps qu'il s'abîme dans son refus de donner son âme, est promis à l'extermination, car, comme il est dit dans la IV ème Ode, il «se retourne désespérément vers la terre ». Tout au contraire, dès l'instant où le héros de Partage accepte, au sens le plus propre, de se convertir, de se lever jusqu'à être exalté "dans la gloire de Dieu ", alors il est promis à la transfiguration.

Nous touchons ici à un mystère profond au sein non seulement de Partage, mais de toute l'œuvre de Claudel : la transfiguration passe par l'extermination, la création par la destruction. La plus saisissante expression de cette dualité se trouve dans la Conversation sur Jean Racine déjà citée et qui, par sa date même et l'une de ses tonalités, prend figure de parole testamentaire :

...A l'origine même de la tragédie [...] le vieil Eschyle dresse la figure grandiose de la prophétesse troyenne [Cassandre], accusatrice de cet Apollon qui l'a séduite: «Apollon! Apollon! Dieu de la porte! mon Apollon de mort! Apôlesas! tu m'as perdue!»

Qu'est-ce à dire, sinon qu'Apollon, père des Muses, Dieu créateur par excellence, est dans le même temps le Dieu destructeur, Dieu « de mort ».

La tragédie antique, et plus près de nous la tragédie classique - même Phèdre - n'ont retenu que la figure de mort; il leur a manqué la transfiguration recréatrice. C'est cette transfiguration que Claudel a saluée dans Rimbaud, le "mystique à l'état sauvage »; c'est elle que lui-même, à sa suite, n'a cessé de traquer d'œuvre en œuvre, frappé dès ses débuts par l'interrogation de Mallarmé : «Qu'est-ce que cela veut dire? » tant il est vrai que toute chose est symbole. Mais il entend, lui, aller plus loin et, à l'écoute de la Bible, passer du symbole à la parabole. Rappelons-nous la phrase, répétée par trois fois dans le Journal et reprise dans Positions et propositions : « Tout ce qui existe est symbole tout ce qui arrive est parabole». Mais tout n'est pas encore proféré. Il faut ajouter : toute parabole est à la fois destruction et création, extermination et transfiguration.

Mon vœu pour ce soir n'était autre que celui-ci : vous entretenir d'abord de l'étrange aventure qui est « arrivée » à Claudel, et vous parler ensuite de la parabole qu'il a su en faire naître sous le titre combien révélateur de son double sens : Partage. - Excusez-moi d'y avoir si incomplètement réussi !

\section{NOTES}

1. Semprun Jorge, L'Ecriture ou la vie, 1994, éditions Gallimard.

2. Lambron Marc, L'EEil du silence, 1993, éditions Flammarion. 
3. Mémoires improvisés.

4. Euvres en prose, Gallimard, Collection la Pléiade.

5. Les états du texte sont publiés dans Partage de Midi, Folio, Théâtre 1994.

6. In Théâtre I, II, Gallimard, Collection la Pléiade.

7. Partage de Midi, Folio, théâtre 1994, p. 257.

8. Euvres en prose, Gallimard, Collection la Pléiade, p. 466. 